\title{
Detection of Skin Cancer using Optical Method
}

\author{
C. Jim Elliot, V. Aarthirani, J. Sivasangari, V. Umasankari, P. Sowmiya
}

\begin{abstract}
Skin is the one of the most important organ present in our body that does some of the important functions like protection, temperature regulation etc. Due to some conditions, skin is affected by many abnormalities. The abnormalities may be smaller or larger, which depends on the damage caused to the skin. Some abnormalities of skin are irritations, allergic reactions, swelling etc. One of the most major problems that affect the skin completely is of skin cancer. Skin cancer is the most common type of cancer that affects the $40 \%$ of people in all over world. It may be caused due to several factors like genetic, working in hazardous chemical areas, exposure to sun, repeated $X$-ray exposures etc. All of these factors affects the skin and causes skin cancer. Skin biopsy is a major technique used at present to detect all types of skin cancers. As this biopsy method is invasive, and takes more time to check the cancer tissue, we are using this optical method for detection of skin cancer. Optical imaging is the upcoming technology that plays the major role in all fields. In this method Near Infra-Red (NIR) light source is passed into the affected skin region. The transimpedance amplifier is used to amplify the output from photodiodes. Then the output from the photodiodes is connected to the Digital Storage Oscilloscope (DSO) in which the waveform shows the variations due to skin cancer.
\end{abstract}

Keywords: Digital Storage Oscilloscope, near infrared light source, Photodiodes, skin cancer.

\section{NEED FOR OPTICAL IMAGING}

$$
\mathrm{T}
$$

The most common type of cancer in human beings is skin cancer where it affects the part or entire region of the skin. There are various types of carcinoma's that affects the skin region partially or completely, they are categorized as Basal cell carcinoma (BCC) and Squamous cell carcinoma (SCC) [4].As per the statistics, the death due to skin cancer has been increasing widely. Finding skin cancer in early stage is critical by the current technique followed by the physicians at present has lot of disadvantages. The technique followed at present is skin biopsy [6]. This technique may cause pain, bleeding, swelling, irritation in patient's body. This skin biopsy

Revised Manuscript Received on December 16, 2019.

* Correspondence Author

C. Jim Elliot*, Department of Biomedical Engineering, Kalasalingam Academy of Research \& Education, VirudhuNagar, India. Email: jimelliot.c@gmail.com

V. Aarthirani, Department of Biomedical Engineering, Kalasalingam Academy of Research \& Education, VirudhuNagar, India. Email: aarthiraniv@gmail.com

V. Umasankari, Department of Biomedical Engineering, Kalasalingam Academy of Research \& Education, VirudhuNagar, India. Email: umasankarivasagaraj@gmail.com

Sowmiya P, Department of Biomedical Engineering, Kalasalingam Academy of Research \& Education, VirudhuNagar, India. Email: sowmiyasowmisp@gmail.com

J. Sivasangari, Department of Biomedical Engineering, Kalasalingam Academy of Research \& Education, VirudhuNagar, India. Email: sangaris559@gmail.com technique requires time and over cost for detecting skin cancer. Hence we come up with the solution called optical imaging for skin cancer detection which overcomes all of the disadvantages in biopsy. This optical method uses near infrared light source for evaluating skin cancer. The infrared light source are subdivided into three major types namely, near infrared light source, mid infrared light source, far infrared light source. Whereas, near infrared light source is used for imaging purpose.

Optical Imaging methods have caused the big evolution in current medical field for lesion detection and discrimination. [1]. Optical imaging is of many types namely, optical tomography, bioluminescence etc [2]. Near infrared source plays a major role in many of fields like neuroimaging, ergonomics, brain computer interface, neonatal research etc. Near infrared light source is harmless and approved by the food and drug administration for continuous usage in human body[3]. Near infrared light source is allowed to pass into the phantom for detecting the abnormalities. The phantom is a tissue mimicking component which has all the properties of the skin used here for testing purpose. The phantom can be prepared from wax, polyethylene, rubber, etc. The chicken pieces are placed inside the phantom where it is assumed as abnormal tissue (cancer affected area). The output from the photodiodes is converted into high level signals by using the transimpedance amplifier.

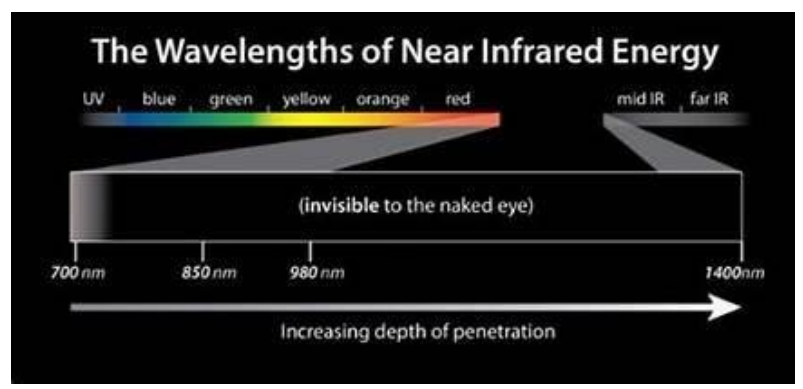

Fig. 1. Electromagnetic spectrum

The transimpedance amplifier is connected to the DSO in order to know the abnormal tissue present inside the phantom. The wavelength of near infrared light source is of 0.7-5 $\mu \mathrm{m}$. The wavelength of mid infrared light source is of 5-40 $\mu \mathrm{m}$. The wavelength of far infrared light source is of $40-300 \mu \mathrm{m}$. 


\section{METHODOLOGY}

\section{A. Near - Infrared Light Source}

Near infrared light source has many advantages hence it plays a major role in current imaging techniques for treating any disease. The penetration of near infrared light source into human skin depends on the chromophores that are presented on the skin. There are various kinds of skin chromophores like melanin, hemoglobin and fat that are present in the skin layers. Chromophores are responsible for the absorption and scattering changes within the human skin, when the skin is irradiated with light source. It allows the penetration deeply into the skin chromophores. It is the kind of non-ionizing radiation and shows high resolution [5] hence specific doses are suggested by the physician can be repeatedly given into the body of the patient.

\section{B. LED Driver Circuit}

The led driver circuit is designed using the near infrared LEDs and battery. The near infrared LEDs are placed in parallel, where the LEDs are provided with the supply of 1.8 volt the circuit is shown in Fig 2. Hence this led driver circuit is used here to pass the near infrared light source into the phantom for detecting the abnormalities.

\section{Photodiodes}

Photodetectors are of two types namely photodiodes and phototransistors. Photodiodes are the kind of diodes that are majorly used for obtaining the electricity from light source. The working principle of photodiodes is based on the photoelectric effect. The photodiodes form electricity by hitting the light signals into the junction. The output from the photodiodes is of low level electrical signals.

\section{Transimpedance Amplifier}

It is the one of the major type of operational amplifier circuits. It is constructed using the IC 741 which is one of the conventional. Indium gas arsenic photodiodes are used here along with $2 \mathrm{k} \Omega$ resistors and $1 \mu \mathrm{F}$ capacitors. The trans impedance amplifier circuit is shown in Fig 3.The entire circuit is used here for the amplification of output from photodiodes which is in the form of low level electrical signals.

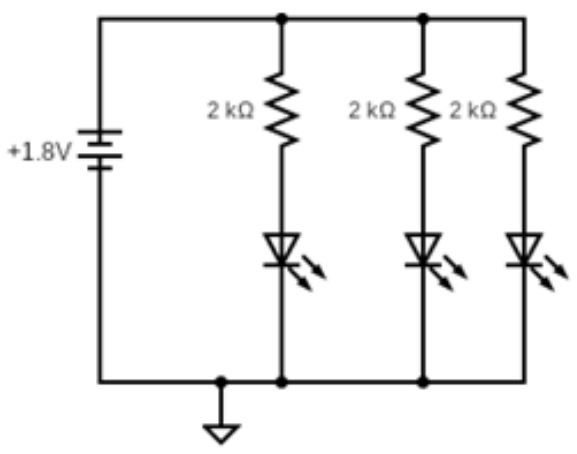

Fig. 2 LED driver circuit

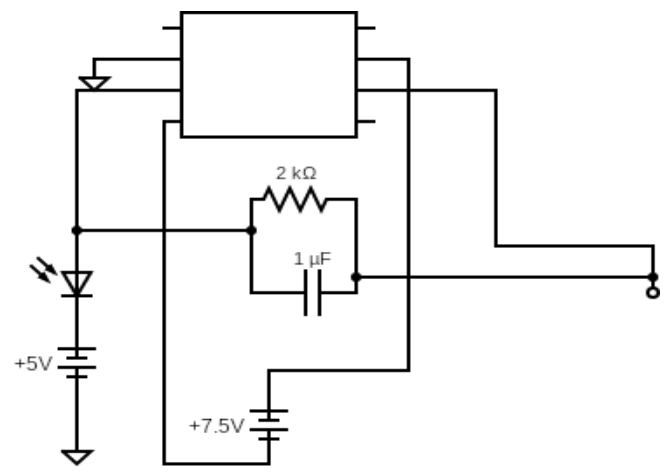

Fig. 3 Transimpedance amplifier circuit

The IC 741 consists of 8 pins, where as in transimpedance amplifier Pin 1, 5 and 8 are not connected. Pin 2 is the inverting pin which is connected to the ground. Pin 3 is non-inverting pin which is connected to the photodiode. Pin 4 and Pin 7 is connected to the battery which provides supply of 7.5 volt. The capacitor and resistor are connected in along with the photodiode. The photodiode is also provided with the supply of 5v. Pin 6 is connected to the DSO.

\section{E. Phantom}

Then the next step involved in the project is to design the phantom using paraffin wax pellets [8] which has tissue mimicking property, whereas the process is shown in Fig 4 The phantom is assumed as the human skin for testing purpose. The pure paraffin wax pellets are collected and placed inside the water bath. The main purpose of using paraffin wax pellets is since it is easily available and can be cast into any desired shape.

\section{PARAFFINWAX PELLETS IS PLACED IN WATERBATH}

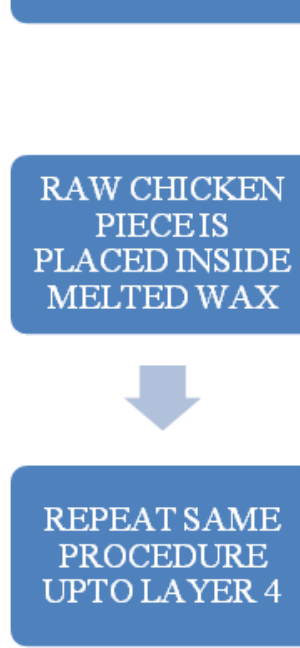

TEMPERATURE $60-70^{\circ} \mathrm{C}$
Fig. 4 Phantom preparation steps

Then the wax pellets are allowed to melt at $60-70{ }^{\circ} \mathrm{c}$. The raw chicken pieces of small sizes are sliced using the knife and placed in the separate container after cleaning and dried. The plastic container where the paraffin wax is placed must be of length $100 \mathrm{~mm}$, breadth $50 \mathrm{~mm}$ and height $10 \mathrm{~mm}$. 
Markings are done in the plastic box, hence it will be useful for making the phantom at correct size. The wax should be stirred well using a glass stirrer. After the wax melts, it should be gently poured into the box. After keeping the melted wax inside the plastic container, the raw chicken piece is placed inside the wax carefully, before it cools. The layer is called as the first layer of the phantom. Then these steps are continued till making the fourth layer of phantom is made. Then the liquid phantom inside the box is allowed to cool for one day. The inclusion placement is mentioned as 1, 2 and 3, in Fig. 5 Then the optical phantom is taken out from the plastic box. The raw chicken pieces placed inside the wax are assumed as abnormal tissue and the places without the chicken pieces are normal region which are not suspected by skin cancer abnormalities. The NIR light source is allowed to pass into this optical phantom, and the photodiodes picks up the signal from the abnormal area where the near infrared light source can't be able to pass. The output from the photodiodes is extracted from this optical phantom. This optical phantom has all of the properties of the human skin.

\section{OUTPUT}

The final waveform produces from normal areas of phantom shows straight line graph which is shown in Fig 6. whereas the waveform obtained from the chicken pieces (cancerous cells) shows some variations which is shown in Fig 7.

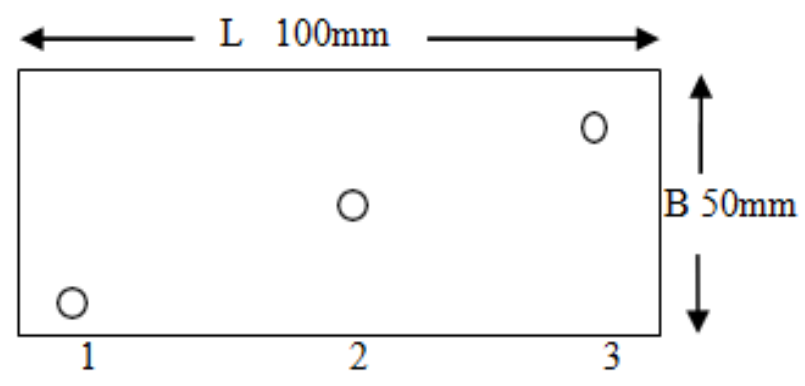

Fig. 5 Inclusion placements in phantom wax

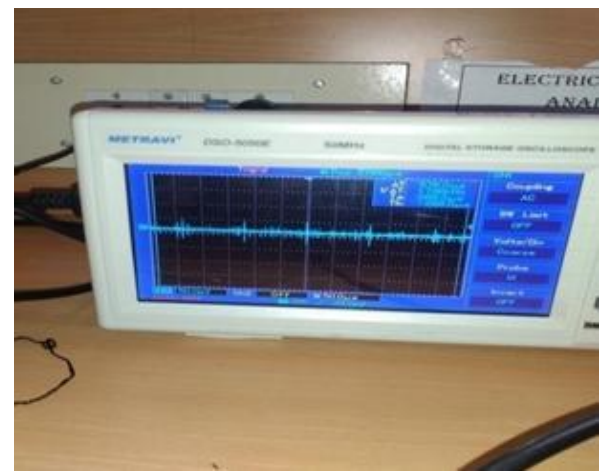

Fig. 6 Normal graph

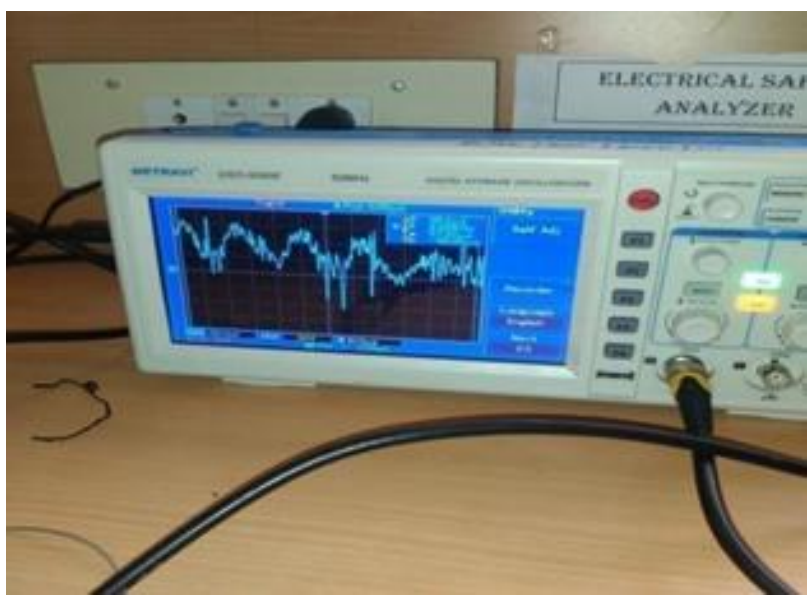

Fig. 7. Abnormal graph

\section{A. Waveform details for normal area}

Hence the time needed by the near infrared light source to penetrate into the normal layer (wax area) is about

$\mathrm{Ta}=$ Initial time needed to penetrate into the tissue $\mathrm{Ta}=288.0 \mu \mathrm{s}$

Whereas, $\mathrm{Tb}=$ final time period to calculated from tissue $\mathrm{Tb}=-288.0 \mu \mathrm{s}$.

By using this $\mathrm{Ta}$ and $\mathrm{Tb}$ values, the change in time $\Delta \mathrm{T}$ is noted as,

$\Delta \mathrm{T}=576.0 \mu \mathrm{s}$ for normal layer of phantom

\section{B. Waveform details for abnormal area}

Hence the time needed for the near infrared light source to penetrate into the phantom is less than that of normal layer because of the blockage that is happened due to the chicken pieces. Hence when near infrared light source is allowed to pass into the phantom it travels and it got blocks due to the presence of this chicken pieces hence there is no way for near infrared light source for further penetration hence the time value will be less than that of normal tissue for penetration. By using this value it is easy to determine the skin cancer.

$\mathrm{Ta}=$ Initial time needed to penetrate into tissue

$\mathrm{Ta}=572.0 \mu \mathrm{s}$

$\mathrm{Tb}=$ final time period calculated from tissue

$\mathrm{Tb}=-58.00 \mu \mathrm{s}$

By using this $\mathrm{Ta}$ and $\mathrm{Tb}$ values the change in time $\Delta \mathrm{T}$ is noted as,

$\Delta \mathrm{T}=115.2 \mu \mathrm{s}$ for abnormal area of phantom

\section{MERITS}

In biopsy, one part of affected tissue are separated from body and evaluated under microscope by the lab technician. It might take up to 2-3 days. The purpose of using optical imaging is it reduces the patient's exposure to harmful radiation and the procedure can be done repeatedly. This method can also be used as a routine screening tool in places where people are working in hazardous / radiation regions.

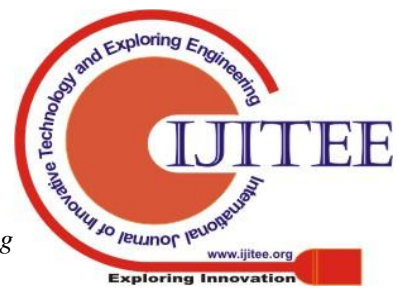




\section{Conclusion}

This optical imaging technique is more efficient when compared to conventional method skin biopsy. The cost involved and time taken for detecting is very less when compared to that of biopsy.

\section{ACKNOWLEDGEMENT}

We would like to thank our mentor Mr. C. Jim Elliot, assistant professor, biomedical engineering department, Kalasalingam academy of research and education for sharing his valuable ideas in this project, and also we would like to thank all the faculty members of our department.

\section{REFERENCES}

1. S.Pratavieria, C.T.Andrade, A.G. Salvio ( 2014,June,13), Optical imaging as auxiliary tool for skin cancer diagnosis. [Paper]. pp. $160-170$

2. Xiaofeng Zhang (2013, March,20).Instrumentation in diffuse optical imaging. [Article]

Available:https://www.researchgate.net/publication/262610713_Instr umentation_in_Diffuse_Optical_Imaging

3. Robert S.Mc Donald (2004,January, 14). A review of infrared spectrometry[Article]

.Available:https://doi.org/10.1021/ac00245a601.

4. T.D.Srividya, V.Arulmozhi (2019, February, 6). A review of threshold based segmentation of skin cancer with image processing. [Journal]. pp 225-227.

5. Abhijit A Gurjarpadhiye, Arita Dubanika, Mansi B Parekh, Jayakumar Rajadas (2015, November,30) .Infrared imaging tools for diagnostic applications in dermatology[Article] pp. 1-5.

6. J.J.Stamena , B.Hamre,L.Zhoo (2017,May,11). Optical detection and monitoring of pigmented skin lesions.[Article]. Available: https://www.ncbi.nlm.nih.gov/pmc/articles/PMC5480441

7. Esperanza Guerra Rosas, Angel coronel Beltran (2015, September,9).A review of threshold based segmentation of skin cancer with image processing. [Journal].pp 225-227

8. R.Srinivasan, D.Kumar and Meghasingh (2002, January, 2). Optical tissue equivalent phantoms for medical imaging [Paper] pp 42-46.

\section{AUTHORS PROFILE}

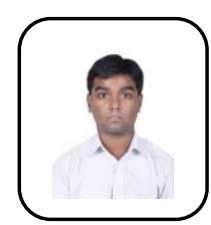

Mr. C. Jim Elliot is working as Assistant Professor in the department of Biomedical Engineering at Kalasalingam Academy of Research \& Education and pursuing his $\mathrm{PhD}$ research in optical imaging. He has worked as Senior Research Fellow in a DST funded project.

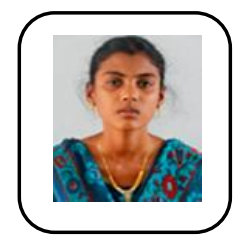

Ms. Umasankari is pursuing B. Tech final year in the stream of biomedical engineering at Kalasalingam Academy of research and education. My father's name is $\mathrm{S}$. Vasagaraj. He is a private employee. My mother's name is M. Amali. She is a homemaker. My strengths are I am a good companion and well decision maker. I had completed my in plant training at Saravana multispeciality hospital for the period of 15 days in Madurai. In that training I had learnt a lot about biomedical equipment's maintenance and calibration inside the hospital. I had also have knowledge about software's like arduino, Java and MATLAB.

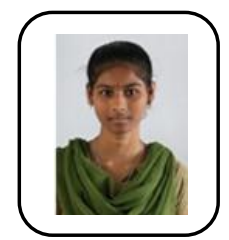

Ms. Sowmiya is pursuing B. Tech in the stream of Biomedical Engineering final year at kalasalingam academy of research and education. My father's name $\mathrm{P}$. Palpandi, he is a mason and my mother's name is $\mathrm{P}$. Sakunthala, she is a milk women. My strengths are time management and I am good in communication. I have practical knowledge and I am a good listener and observer, workaholic too. I had completed my in-plant training at meenakshi mission hospital and research center for the period of 14 days at Madurai. In that training I gained more knowledge about biomedical equipment's, service, maintenance, calibration, and biomedical engineer's responsibilities and their roles towards the hospital.

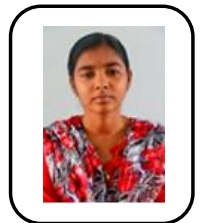

Ms. J. Sivasangari is pursuing B. Tech final year in the stream of Biomedical engineering at Kalasalingam Academy of research and education. My father's name is B. Jeyakannan, he is working as a private employee. My mother's name is P. Geetha, she is working as a teacher in government school. My strengths are I am an optimist and well decision maker. I had completed type writing course in Tamil and English languages. I also have basic knowledge about software's like C and Java. I had completed my in-plant training at Saravana multi speciality hospital for the period of 15days at Madurai.

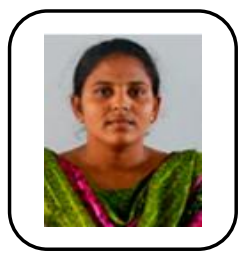

Ms. V.Aarthirani. is pursuing B. Tech final year in the stream of biomedical engineering at Kalasalingam academy of research and education. My Father's name is V. Vellaidurai, he is a ex-army man. My mother's name is V. Amutha, she is a home maker. I had completed my in-plant training at Saravana multi speciality hospital, Madurai for the period of 15 days. 